\title{
Estimating $\varepsilon^{\prime} / \varepsilon$ in the standard model independently of $\operatorname{Im} \lambda_{t}$
}

\author{
Marco Fabbrichesi \\ INFN, Sezione di Trieste and \\ Scuola Internazionale Superiore di Studi Avanzati \\ via Beirut 4, I-34013 Trieste, Italy.
}

(November 18, 2018)

\begin{abstract}
The $C P$-violating parameter $\varepsilon^{\prime} / \varepsilon$ is estimated in a novel way by including the explicit computation of $\varepsilon$ in the ratio as opposed to the usual procedure of taking its value from the experiments. This approach has the advantage of being independent from the determination of the CKM parameters $\operatorname{Im} \lambda_{t}$ and of showing more directly the dependence on the long-distance parameters. The matrix elements are taken from the chiral quark model approach, the parameters of which have been recently determined by an updated fit of the $C P$-conserving amplitudes entering the $\Delta I=1 / 2$ rule. By sampling the ranges of the experimental inputs according to a normal distribution and those of the theoretical uncertainties according to a flat one, it is found that
\end{abstract}

$$
\varepsilon^{\prime} / \varepsilon=(2.5 \pm 0.9) \times 10^{-3},
$$

in agreement with the current experimental data. A more conservative estimate

$$
0.9 \times 10^{-3}<\varepsilon^{\prime} / \varepsilon<5.8 \times 10^{-3}
$$

is found by considering the smallest and the largest values obtained by taking a flat distribution for all inputs.

In the standard model, the $C P$-violating parameter $\varepsilon^{\prime} / \varepsilon$ can be in principle different from zero because the $3 \times 3$ Cabibbo-Kobayashi-Maskawa (CKM) matrix $V_{i j}$, which appears in the weak charged currents of the quark mass eigenstate, is in general complex. On the other hand, in other models like the superweak theory [1], the only source of $C P$ violation resides in the $K^{0}-\bar{K}^{0}$ mixing, and $\varepsilon^{\prime}$ vanishes. Establishing the precise value of $\varepsilon^{\prime}$ is therefore of great importance.

Experimentally $\varepsilon^{\prime} / \varepsilon$ is extracted, by collecting $K_{L}$ and $K_{S}$ decays into pairs of $\pi^{0}$ and $\pi^{ \pm}$, from the relation

$$
\operatorname{Re} \varepsilon^{\prime} / \varepsilon \simeq\left[\left|\eta_{+-} / \eta_{00}\right|^{2}-1\right] / 6,
$$

and the determination of $\eta_{00}$ and $\eta_{+-}$which are, respectively, the ratio of the amplitudes for $K_{L} \rightarrow \pi^{0} \pi^{0}$ over $K_{S} \rightarrow \pi^{0} \pi^{0}$ and $K_{L} \rightarrow \pi^{+} \pi^{-}$over $K_{S} \rightarrow \pi^{+} \pi^{-}$.

With the announcement last year of the preliminary result from the KTeV collaboration (FNAL) [2] based on data collected in 1996-97, and from the NA48 collaboration (CERN) [3] based on data collected in 1997-98, the longstanding issue of whether $\varepsilon^{\prime}$ vanishes or not seems to be settled. By computing the average (see Fig. 3 below) among the two 1992 experiments (NA31 [迎] and E731 [5]) and the preliminary data of KTeV and NA48 one obtains

$$
\operatorname{Re} \varepsilon / \varepsilon^{\prime}=(1.9 \pm 0.46) \times 10^{-3} .
$$

The error in (2) has been inflated according to the Particle Data Group procedure for combining results with substantially different central values. The value and range in (2) can be considered the current experimental result. Such a result will be further improved by the complete run and full data analysis from KTeV and NA48 and the first data from KLOE (Frascati) thus achieving an uncertainty of only few parts in $10^{4}$.

From the theoretical point of view, $\varepsilon^{\prime}$, which parameterizes direct $C P$ violation in the decays (for a review see, e.g., [6] 8]), is computed as

$$
\varepsilon^{\prime}=\frac{G_{F} \omega}{2 \operatorname{Re} A_{0}} \operatorname{Im} \lambda_{t}\left[\Pi_{0}-\frac{1}{\omega} \Pi_{2}\right],
$$

where $\operatorname{Im} \lambda_{t} \equiv \operatorname{Im} V_{t d} V_{t s}^{*}$ 


$$
\begin{aligned}
& \Pi_{0}=\frac{1}{\cos \delta_{0}} \sum_{i} y_{i} \operatorname{Re}\left\langle Q_{i}\right\rangle_{0}\left(1-\Omega_{\eta+\eta^{\prime}}\right), \\
& \Pi_{2}=\frac{1}{\cos \delta_{2}} \sum_{i} y_{i} \operatorname{Re}\left\langle Q_{i}\right\rangle_{2},
\end{aligned}
$$

and $\left\langle Q_{i}\right\rangle_{I}=\left\langle 2 \pi, I\left|Q_{i}\right| K\right\rangle$. The phases $\delta_{0,2}$ come from final state interactions. The explicit forms of $\Delta S=1$ four-quark operators $Q_{i}$ can be found, for instance, in [8]. The term $\Omega_{\eta+\eta^{\prime}}$ is the isospin breaking (for $m_{u} \neq m_{d}$ ) contribution of the mixing of $\pi$ with $\eta$ and $\eta^{\prime}$. In (3), the phase $\phi=\pi / 2+\delta_{0}-\delta_{2}-\theta_{\epsilon}=(0 \pm 4)^{0}$ has been taken as vanishing [9], and $C P T$ is assumed to hold. The Wilson coefficients $y_{i}$ are known to the next-to-leading (NLO) order in $\alpha_{s}$ and $\alpha_{w}$ [10]. Notice the explicit final-state-interaction phases $\delta_{I}$ which comes from writing in (位) and (5) the absolute values of the amplitudes in term of their dispersive parts.

The parameter $\varepsilon$, which parameterizes indirect $C P$ violation, is given by

$$
\varepsilon=\operatorname{Im} \lambda_{t} C_{\varepsilon} \hat{B}_{K}\left\{\operatorname{Re} \lambda_{c}\left[\eta_{1} S_{0}\left(x_{c}\right)-\eta_{3} S_{1}\left(x_{c}, x_{t}\right)\right]-\operatorname{Re} \lambda_{t} \eta_{2} S_{0}\left(x_{t}\right)\right\}
$$

where $\lambda_{c}=V_{c d} V_{c s}^{*}, S_{i}(x)$ are the Inami-Lim functions [11], $x_{i}=m_{i}^{2} / m_{W}^{2}$ and $C_{\varepsilon}$ is a constant equal to $G_{F}^{2} f_{K}^{2} m_{K} m_{W}^{2} /\left(3 \sqrt{2} \pi^{2} \Delta m_{L S}\right) . \quad \hat{B}_{K}$ is the bag parameter to be determined in the long-distance estimate of the hadronic matrix element for the $\Delta S=2$ transition. In (6), a term of higher order in $\lambda \equiv V_{u s}$ is neglected. The parameters $\eta_{i}$, which encode the renormalization group running, are known to the NLO order [12].

In the usual approach - followed by all current estimates - the ratio $\varepsilon^{\prime} / \varepsilon$ is computed by dividing (3) by the experimental value of $\varepsilon$. The allowed values for the CKM combination $\operatorname{Im} \lambda_{t}$ in front of (3) are then either taken from independent analysis of the unitarity triangle 13 15] or consistently computed by means of a determination of $\varepsilon$ in the same model (which includes the long-distance matrix element of the $\Delta S=2$ amplitudes) 16 18].

This two-step procedure can be by-passed by computing in a given model $\varepsilon^{\prime}$ and $\varepsilon$ and by taking directly their ratio. Because both parameters are proportional to $\operatorname{Im} \lambda_{t}$, this quantity, which is an important source of uncertainty, simplifies in the ratio. This approach has also been advocated in 19.16] without, however, any numerical analysis. Of course, one trades the dependence on the Wolfenstein parameter $\eta$ for that on $\rho$ in the factor $\operatorname{Re} \lambda_{t}=-\lambda(1-$ $\left.\lambda^{2} / 2\right) V_{c b}^{2}(1-\bar{\rho})$ that enters in (6). However, this dependence is weaker and $\bar{\rho} \equiv\left(1-\lambda^{2} / 2\right) \rho$ can be determined reasonably well in the unitarity triangle independently of kaon physics. Another advantage of this procedure is that the dependence of the final result on many of the input variables is made more transparent because the computation is not separated into the determination of $\operatorname{Im} \lambda_{t}$ on the one hand and $\varepsilon^{\prime} / \varepsilon$ on the other. However, as we shall see, the uncertainty in the final estimate is about the same size as that obtained by the usual method, and it is still dominated by the uncertainty in the hadronic matrix elements.

In this work I use this second approach and apply it to the estimate of $\varepsilon^{\prime} / \varepsilon$ in the standard model.

The hadronic matrix elements for all the relevant quark operators $Q_{1-10}$ and the parameter $\hat{B}_{K}$ are computed in the chiral quark model $(\chi \mathrm{QM})$ 20,21] at $O\left(p^{4}\right)$ in the chiral expansion along the lines presented in 18. I use this approach because it is the only theoretical model in good agreement with the experimental data.

As we shall see, the final result is consistent with that of the $\chi \mathrm{QM}$ obtained by the usual method.

In the $\chi \mathrm{QM}$ approach, there are three model-dependent parameters $M,\langle\bar{q} q\rangle \equiv(\langle\bar{q} q\rangle)^{1 / 3}$ and $\left\langle\alpha_{s} G G / \pi\right\rangle$ which are fixed by means of a fit of the $C P$-conserving amplitudes in the $\Delta I=1 / 2$ selection rule of $K \rightarrow \pi \pi$ decays. This fit has been re-done recently in [22] by updating those short-distance inputs that have become better known in the meantime. The matching of the hadronic matrix elements to the Wilson coefficients is done at the scale $\mu=0.8$ $\mathrm{GeV}$. The amplitudes $A\left(K^{0}-2 \pi, I=0\right)$ and $A\left(K^{0}-2 \pi, I=2\right)$ computed in the model are compared with their experimental values by allowing at most a $\pm 20 \%$ uncertainty. The values for $M,\langle\bar{q} q\rangle$ and $\left\langle\alpha_{s} G G / \pi\right\rangle$ found are those in Table I. The values for the two condensates vary according to the $\gamma_{5}$-scheme of dimensional regularization, 't Hooft-Veltman (HV) or Naive Dimensional Regularization (NDR), used in the computation of the NLO Wilson coefficients and hadronic matrix elements.

Having thus determined the model-dependent parameters, it is possible to compute $\varepsilon^{\prime} / \varepsilon$.

Notice that the uncertainty in $\Omega_{\eta+\eta^{\prime}}$ [23] affects the final estimate only marginally since any change in this input implies a change in the fit to the $\Delta I=1 / 2$ rule with the net effect of recovering the original prediction. In fact, any change in $\Omega_{\eta+\eta^{\prime}}$ is anti-correlated to the value of $\left\langle\alpha_{s} G G / \pi\right\rangle$ oobtained in the fit, the variation of which compensates in $\varepsilon^{\prime} / \varepsilon$ the original change of $\Omega_{\eta+\eta^{\prime}}$. Changing the value of $m_{c}$ affects the value of $\varepsilon^{\prime} / \varepsilon$ below the $10 \%$ level.

The value of $\varepsilon^{\prime} / \varepsilon$ depends - besides other given parameters like meson masses and decay constants, and the matching scale $\mu$ that we keep fixed - on eight input parameters: $\mathrm{M},\langle\bar{q} q\rangle,\left\langle\alpha_{s} G G / \pi\right\rangle, m_{s}, \Lambda_{\mathrm{QCD}}, m_{t}, V_{c b}$ and $\bar{\rho}$. The range for $\bar{\rho}$ is that obtained in the unitarity triangle without the bounds from the experimental value of $\varepsilon$. See Table I for a summary of the ranges used. For central values of all input parameters, $\varepsilon^{\prime} / \varepsilon$ is equal to $2.5 \times 10^{-3}$ in both $\gamma_{5}$-schemes. This value corresponds to the determination $\hat{B}_{K}=1.0$. By comparison with the experimental value of $\varepsilon$, it is then found that $\operatorname{Im} \lambda_{t}=1.3 \times 10^{-4}$ and $1.2 \times 10^{-4}$, respectively, in the HV and NDR scheme. 


\begin{tabular}{|c|c|}
\hline parameter & value \\
\hline$\Lambda_{\mathrm{QCD}}^{(4)}$ & $340 \pm 40 \mathrm{MeV}$ \\
\hline$\left|V_{c b}\right|$ & $0.0405 \pm 0.0015[24]$ \\
\hline $\bar{\rho}$ & $0.242_{-0.045}^{+0.070} 24$ \\
\hline $\bar{m}_{t}\left(m_{t}\right)$ & $165 \pm 5 \mathrm{GeV}$ \\
\hline$\Omega_{\eta+\eta^{\prime}}$ & $0.25 \pm 0.10$ \\
\hline $\bar{m}_{s}(1 \mathrm{GeV})$ & $150 \pm 25 \mathrm{MeV}$ \\
\hline$\langle\bar{q} q\rangle$ & HV: $(-210 \leftrightarrow-260 \mathrm{MeV})^{3}$, NDR: $(-230 \leftrightarrow-260 \mathrm{MeV})^{3}$ \\
\hline$\left\langle\alpha_{s} G G / \pi\right\rangle$ & HV: $(325 \leftrightarrow 335 \mathrm{MeV})^{4}$, NDR: $(327 \leftrightarrow 340 \mathrm{MeV})^{4}$ \\
\hline$M$ & $\mathrm{HV}: 180 \leftrightarrow 220 \mathrm{MeV}, \mathrm{NDR}: 185 \leftrightarrow 210 \mathrm{MeV}$ \\
\hline
\end{tabular}

TABLE I. Table of the numerical values of the most relevant input parameters used in the present analysis.

In order to estimate the error, a set of values for $\varepsilon^{\prime} / \varepsilon$ is generated by varying the parameters $M,\left\langle\alpha_{s} G G / \pi\right\rangle,\langle\bar{q} q\rangle$ in a flat scanning of their given ranges, while the others according to a normal distribution. The difference in the values of $\varepsilon^{\prime} / \varepsilon$ found in the HV and NDR schemes is rather small in the $\chi \mathrm{QM}$ approach and it can be considered as part of the overall theoretical uncertainty by joining the values found in the two schemes. The set of values of $\varepsilon^{\prime} / \varepsilon$ thus found is amenable of statistical analysis. For a given set, a distribution is obtained by collecting the values of $\varepsilon^{\prime} / \varepsilon$ in bins of a given range. This is shown in Fig. 1. The final distribution is partially skewed, with more values closer to the lower end but a longer tail toward larger values.

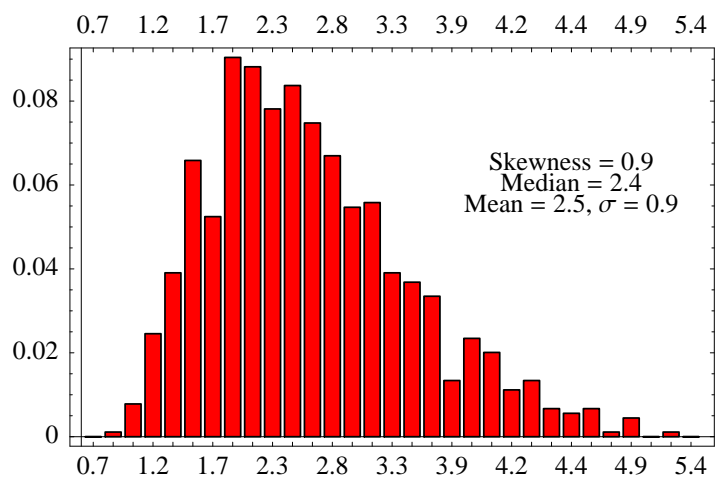

FIG. 1. Distribution of values of $\varepsilon^{\prime} / \varepsilon$. Normalized bins versus the central values $\left(\times 10^{3}\right)$ of each bin.

However, because the skewness in the distribution is less than one (and the standard deviation of the variance much smaller than the variance itself), the mean and the standard deviation are a good estimate of the central value and the dispersion of values around it. The statistical analysis therefore yields

$$
\varepsilon^{\prime} / \varepsilon=(2.5 \pm 0.9) \times 10^{-3},
$$

which is the best estimate quoted in the abstract.

The $1 \sigma$ uncertainty of the estimate (7) should be considered together with the range given by the largest and the smallest values of $\varepsilon^{\prime} / \varepsilon$ when all input parameters are varied in a flat scanning within their errors. In this case, it is found that

$$
0.9 \times 10^{-3}<\varepsilon^{\prime} / \varepsilon<5.8 \times 10^{-3},
$$

which is the more conservative result.

It is also possible to gain some insight on the dependence of these results on the input parameters. For instance, in Fig. 2, the generated values of $\varepsilon^{\prime} / \varepsilon$ are plotted against, respectively, $\langle\bar{q} q\rangle$ and $m_{s}$ while randomly varying the other parameters. In particular, larger values of $\langle\bar{q} q\rangle$ correspond to larger values of $\varepsilon^{\prime} / \varepsilon$.

There is no strong dependence on $m_{s}$ or $\left\langle\alpha_{s} G G / \pi\right\rangle$ because $m_{s}$ only enters in the NLO corrections and the gluon consensate does not contribute to the leading penguin operators. However, these two parameters play a role in the determination of $\varepsilon$ where the final values of $\hat{B}_{K}$ depends on $m_{s}$ and $\left\langle\alpha_{s} G G / \pi\right\rangle$. Given (6), $\varepsilon^{\prime} / \varepsilon$ is inversely proportional to the value of $\hat{B}_{K}$. 

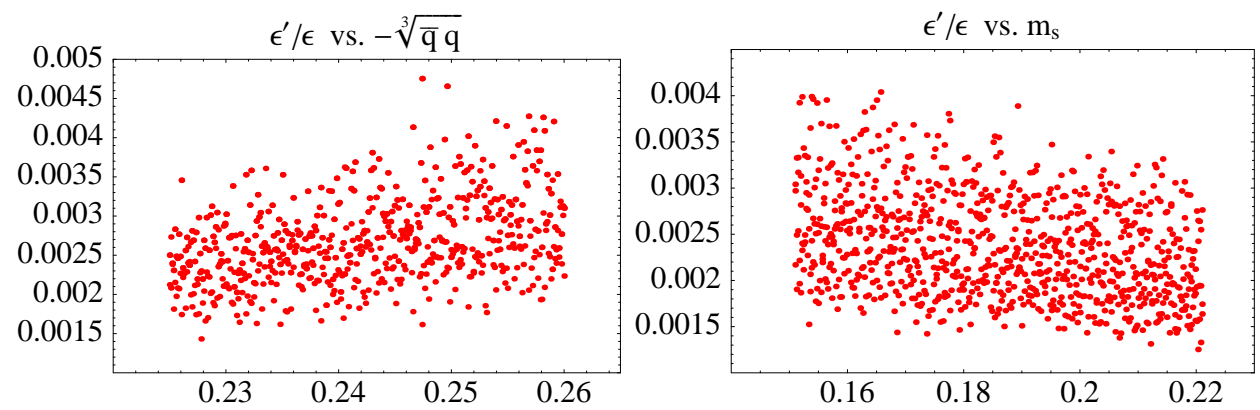

FIG. 2. Variations of $\varepsilon^{\prime} / \varepsilon$ as the quark condensate $\langle\bar{q} q\rangle$ and $m_{s}$ are varied. Other inputs are randomly generated within their bounds.

The results in (7) and (8) are depicted in Fig. 3 as yellow/light-gray and gray bands together with the most recent experimental determinations and the average in (2). As it can be seen from this figure, the result is in good agreement with the experimental data.

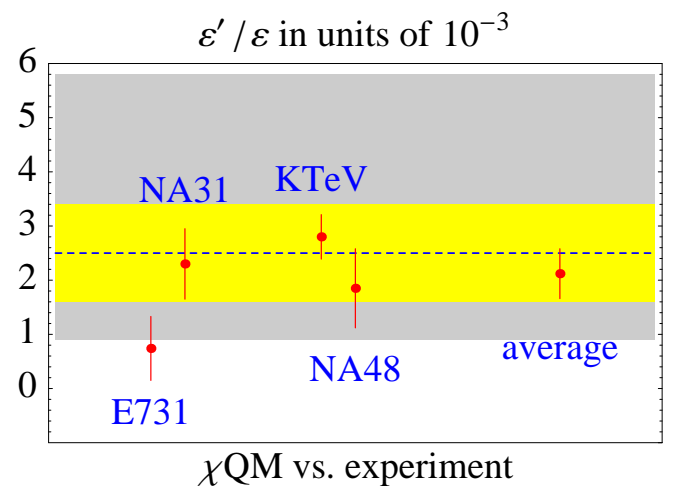

FIG. 3. Experiments vs. the present estimate of $\varepsilon^{\prime} / \varepsilon$. The dashed line is the central value, the gray area spans the conservative range (\$) and the yellow/light-gray area spans the $1 \sigma$ range in (đ).

As discussed in more details somewhere else [22], the rather large values of $\varepsilon^{\prime} / \varepsilon$ found by means of the $\chi \mathrm{QM}$ hadronic matrix elements, and the corresponding better agreement with the experiments, comes about because the fit of the $\Delta I=1 / 2$ rule - on which the approach is based - enhances the contribution of the isospin $I=0$ channel which, in turns, drives $\varepsilon^{\prime} / \varepsilon$ toward larger values.

I thank my collaborators S. Bertolini and J. Eeg for discussions and F. Parodi and A. Stocchi for their help in the determination of $\bar{\rho}$.

[1] L. Wolfenstein, Phys. Rev. Lett. 13 (1964) 562.

[2] KTeV Collaboration (A. Alavi-Harati et Al.), Phys. Rev. Lett. 83 (1999) 22.

[3] NA48 Collaboration (V. Fanti et al.), Phys. Lett. B465 (1999) 335 and A. Ceccucci's talk, CERN February 29, 2000 (http://www.cern.ch/NA48/Welcome.html).

[4] NA31 Collaboration (G. D. Barr et Al.), Phys. Lett. B317 (1993) 233.

[5] E731 Collaboration (L. K. Gibbons et Al.), Phys. Rev. D55 (1997) 6625.

[6] B. Winstein and L. Wolfenstein, Rev. Mod. Phys. 65 (1993) 1113.

[7] G. Buchalla and A. J. Buras and M. E. Lautenbacher, Rev. Mod. Phys. 68 (1996) 1125.

[8] S. Bertolini, J. Eeg and M. Fabbrichesi, Rev. Mod. Phys. 72 (2000) 65.

[9] L. Maiani and G. Pancheri and N. Paver, The DAPHNE Physics Handbook (Frascati, 1992). 
[10] A. J. Buras et AL, Nucl. Phys. B370 (1992) 69 and Nucl. Phys. B400 (1993) 37;

A. J. Buras, M. Jamin and M.E. Lautenbacher, Nucl. Phys. B400 (1993) 75 and Nucl. Phys. B408 (1993) 209; M. Ciuchini, E. Franco, G. Martinelli and L. Reina, Nucl. Phys. B415 (1994) 403.

[11] T. Inami And C. S. Lim, Prog. Theor. Phys. 65 (1981) 297.

[12] A. J. Buras And M. Jamin And P. H. Weisz, Nucl. Phys. B347 (1990) 491;

S. Herrlich And U. Nierste, Phys. Rev. D52 (1995) 6505 and Nucl. Phys. B476 (1996) 27.

[13] T. HambYe eT AL., hep-ph/9906434.

[14] A. A. BEL'KOV ET AL., hep-ph/9907335.

[15] H.-Y. Cheng, hep-ph/9911202.

[16] S. Bosch ET AL., hep-ph/9904408.

[17] M. Ciuchini et al., hep-th/9910236.

[18] S. Bertolini, J. Eeg And M. Fabbrichesi, Nucl. Phys. B514 (1998) 63 and 93.

[19] G. C. Branco et Al., CP Violation (Oxford Science Publications, Oxford 1999).

[20] K. Nishijima, N. Cim. 11 (1959) 698;

F. Gursey, N. Cim. 16 (1960) 23 and Ann. Phys. 12 (1961) 91;

J.A. Cronin, Phys. Rev. 161 (1967 1483;

S. Weinberg, Physica 96A (1979) 327;

A. Manohar And H. Georgi, Nucl. Phys B234 (1984) 189;

A. Manohar And G. Moore, Nucl. Phys B243 (1984) 55;

D. Espriu et AL., Nucl. Phys B345 (1990) 22.

[21] A. Pich and E. De Rafael, Nucl. Phys B358 (1991), 311.

[22] S. Bertolini, J. Eeg And M. Fabbrichesi, hep-ph/0002234

[23] For a recent discussion, see, e.g., S. Gardner And G. Valencia, Phys. Lett. B466 (1999) 355.

[24] F. Parodi, P. Roudeau and A. Stocchi, N. Cim. A112 (1999) 833. 